\title{
Review of: "Combining higher accumulation of amylopectin, lysine and tryptophan in maize hybrids through genomics-assisted stacking of waxyl and opaque2 genes"
}

\author{
Yogesh Vikal ${ }^{1}$ \\ 1 Punjab Agricultural University
}

Potential competing interests: The author(s) declared that no potential competing interests exist.

The article focuses on product development by pyramiding 02 and wx gene using genomics assisted breeding that has significance both in food-chain and different industrial applications. The study was systematically planned and executed. Detailed analysis was performed in terms of foreground, background and phenotypic selection. Agronomic performance of derived lines and hybrids was evaluated in multilocation trails. The developed waxy hybrids had high amylopectin content and also rich in lysine and tryptophan. These two essential amino acids are not synthesized in our body, thus possess paramount importance for growth and development in humans and due to increase accumulation of amlopectin, waxy maize is easily digested in the human gut. Waxy maize grain with essential amino acids can be a major ingredient of high-energy feeds for livestock. 